\title{
Experiencia quirúrgica del Servicio de Otorrinolaringología y Cirugía de Cabeza y Cuello del Hospital Guillermo Grant Benavente durante el periodo 2007-2011
}

\section{Surgical experience of the Department of Otolaryngology and Head and Neck Surgery from Guillermo Grant Benavente Hospital}

David Astudillo 0', Joaquín Ulloa S², Patricio Ulloa B'.

\begin{abstract}
RESUMEN
Introducción: Nuestra especialidad plantea una amplia gama de polos de perfeccionamiento medicoquirúrgico. Es necesario conocer la situación actual de nuestros servicios, en base a lo cual poder delinear futuras áreas de desarrollo.

Objetivo: Conocer la experiencia quirúrgica del Servicio de Otorrinolaringología y Cirugía de Cabeza y Cuello del Hospital Guillermo Grant Benavente.

Material y método: Estudio observacional retrospectivo. Análisis de libros de pabellón, protocolos quirúrgicos e información estadística del centro durante los años 2007-2011.

Resultados: Se realizaron 8.262 cirugías durante el periodo estudiado, con un promedio de 1.921 cirugías anuales. Las áreas quirúrgicas más prevalentes son faringe (44\%), rinosinusología (18\%) y otología (17\%).

Discusión: En nuestro centro se desarrolla una amplia y variada gama de procedimientos quirúrgicos. Existen áreas de un significativo potencial de desarrollo, tales como la cirugía endoscópica rinosinusal y la cirugía de cabeza y cuello.
\end{abstract}

Palabras clave: Experiencia quirúrgica, cirugía otorrinolaringológica.

\begin{abstract}
Introduction: Our specialty poses a wide range of medical and surgical areas of improvement. We need to know the current status of our services to delineate future development areas.

Aim: To determine the surgical experience of the Department of Otolaryngology and Head and Neck Surgery from Guillermo Grant Benavente Hospital.

Material and method: Retrospective observational study. Surgery room books, surgical protocols and statistical information center reviews.

Results: We performed 8262 surgeries during the study period, with an average of 1921 surgeries annually. The most prevalent surgical areas are Pharynx (44\%), Rhinosinusal (18\%) and Otology (17\%).
\end{abstract}

\footnotetext{
Médico de Otorrinolaringología, Hospital Guillermo Grant Benavente, Universidad de Concepción.

2 Interno de Medicina, Universidad de Concepción.
} 
Conclusions: In our center, we developed a wide and varied range of surgical procedures. There are areas of significant development potential, such as endoscopic rhinosinusal and head and neck surgery.

Key words: Surgical experience, otolaryngology surgery.

\section{INTRODUCCIÓN}

Si bien existen registros indios de cirugías de reconstrucción de nariz, labios y pabellón auricular que datan de $2500 \mathrm{AC}$, la otorrinolaringología no se constituyó como especialidad médica sino hasta inicios del siglo XIX, estando sus albores y apogeo inicial íntimamente vinculados al desarrollo de la endoscopía ${ }^{1,2}$. Comprende el conjunto de los conocimientos teórico-prácticos necesarios para la prevención, el diagnóstico y tratamiento médicoquirúrgico de la patología infecciosa, oncológica, traumática, congénita y degenerativa del oído, fosas y senos paranasales, faringe y laringe, así como el manejo médico-quirúrgico de la patología de cabeza y cuello.

Conforme su desarrollo la ha convertido en una especialidad adulta, hemos visto sus límites constantemente redefinidos, alcanzando progresivamente un mayor impacto en el quehacer médico-quirúrgico; es así como el desarrollo y la aplicación médica de nuevas tecnologías en áreas diagnósticas y terapéuticas, tanto más quirúrgicas, amplían en forma exponencial los alcances de nuestra especialidad, abarcando hoy por hoy no tan sólo las áreas tradicionalmente descritas, sino también compartiendo aquellas previamente reservadas para el quehacer neuroquirúrgico, oftalmológico, plástico y oncológico, proveyendo vías de abordaje diagnóstico-terapéutico y técnicas de mayor precisión y menor invasividad en pro del bienestar de nuestros pacientes.

En este contexto, creemos una obligación de todo médico otorrinolaringólogo y de todo centro de otorrinolaringología, el ser partícipe y protagonista del desarrollo y expansión de la especialidad, resultando imperioso para este fin, iniciar con el diagnóstico de la situación actual de cada uno de nuestros centros, planificando a partir de esto las potenciales áreas de desarrollo que debamos explorar.

Bajo este precepto, el presente trabajo pretende conocer y presentar la experiencia quirúrgica del Servicio de Otorrinolaringología y Cirugía de Cabeza y Cuello del Hospital Guillermo Grant Benavente durante el periodo comprendido entre enero de 2007 y abril de 2011, establecer el rendimiento quirúrgico de nuestro servicio respecto al de los demás estamentos de nuestro centro y determinar la importancia relativa de las áreas quirúrgicas más prevalentes dentro del universo de procedimientos realizados.

\section{MATERIAL Y MÉTODO}

Estudio observacional de tipo retrospectivo. Los datos analizados fueron obtenidos en base a la información contenida en los libros de pabellón del Servicio de Otorrinolaringología y Cirugía de Cabeza y Cuello correspondientes al periodo comprendido entre 01.01 .2007 y 30.04.2011, corroborada y complementada mediante el análisis de protocolos quirúrgicos y fichas clínicas, así como también con la información provista por el Departamento de Estadística del Hospital Guillermo Grant Benavente. No se incluyen en el presente estudio las cirugías realizadas por el departamento de base de cráneo, puesto que administrativamente, éstas, si bien son realizadas por un equipo mixto otorrinolaringológico-neuroquirúrgico, son codificadas por el Servicio de Neurocirugía. Asimismo, las cirugías realizadas por el Departamento de Cabeza y Cuello, aun cuando en nuestro hospital son realizadas por otorrinolaringólogos desde hace aproximadamente 5 años, administrativamente codifican como cirugía otorrinolaringológica sólo a partir de marzo de 2011, por lo que aquellas realizadas con anterioridad no son incluidas en el presente estudio. Los datos fueron registrados y analizados en planilla Microsoft Office Excel 2007 y Statistical Package for the Social Sciences 17.

\section{RESULTADOS}

Durante el periodo de estudio, se realizaron en nuestro servicio un total de 8.262 cirugías, con un 
promedio de 1.921 intervenciones anuales, es decir, 158,8 cirugías mensuales. Figura 1.

Al analizar el rendimiento quirúrgico, se aprecia un incremento progresivo del número de cirugías mensuales durante el periodo de enero 2007 a enero 2010; es posible apreciar como el rendimiento quirúrgico cae en febrero de 2010 y se retoma en noviembre del mismo año. Figura 2.

El total de procedimientos descritos corresponden al 6,3\% de las cirugías realizadas en nuestro centro hospitalario durante el periodo señalado, alcanzando un índice de 3,02 días cama utilizados por cada intervención. Durante el mismo periodo, el índice alcanzado por los demás servicios de nuestro hospital fue de 12,7 días cama por cada cirugía.

Dentro del total de intervenciones quirúrgicas realizadas, las áreas que presentaron una mayor prevalencia correspondieron a faringe (44\%), rinosinusología $(18 \%)$ y otología $(17 \%)$. Del total de patología rinosinusal, el $33 \%$ fue resuelta por abordaje endoscópico.

Al establecer un análisis de la evolución temporal de las distintas áreas quirúrgicas, se aprecia un

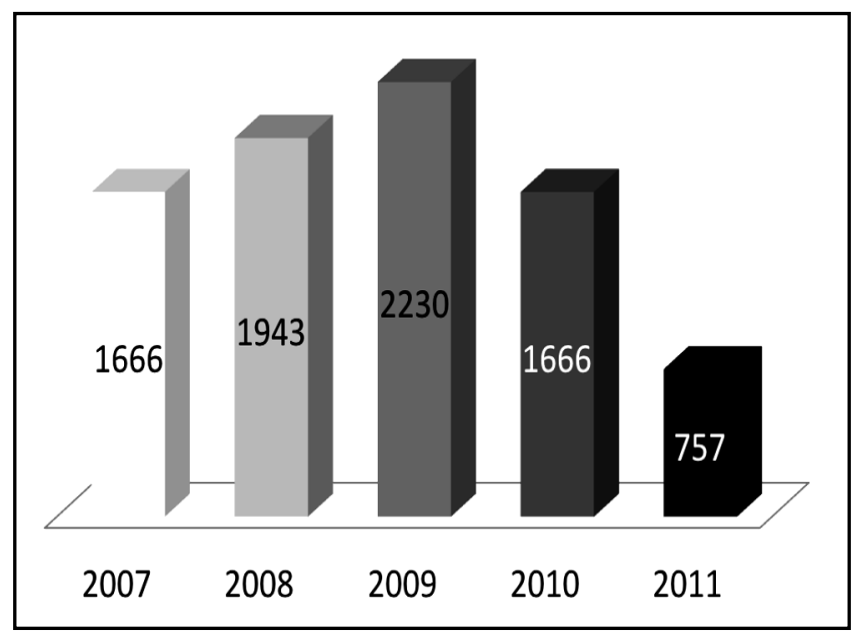

Figura 1. Cirugías anuales.

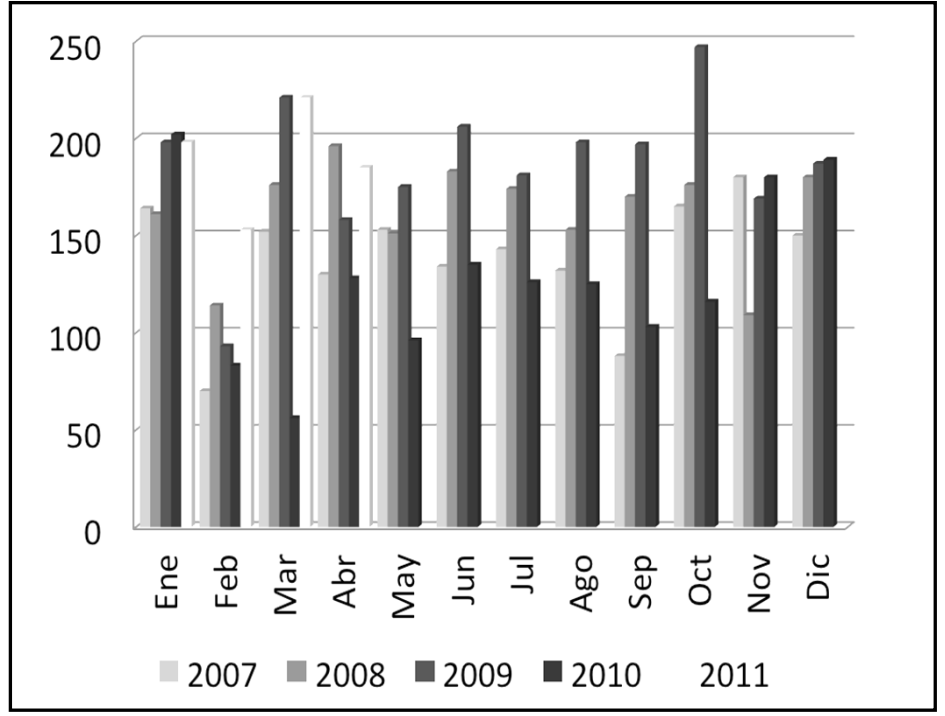

Figura 2. Rendimiento quirúrgico mensual. 
descenso progresivo de la cirugía de oído y faringe, en contraposición de un incremento de la cirugía rinosinusal, principalmente endoscópica, y de cabeza y cuello. Figura 3.

Respecto a la cirugía de oído, se realizaron un total de 1.410 intervenciones, de la cuales 367 (17\%) correspondieron a timpanoplastías y 159 $(11 \%)$ a cirugías radicales.

En relación a la cirugía rinosinusal, 1.017 fueron realizadas por abordaje tradicional, entre las cuales $254(25 \%)$ correspondieron a septoplastías y $179(17 \%)$ a rinoseptoplastías, las cuales se desarrollaron en su mayoría por técnica abierta. Por su parte, de las 463 intervenciones realizadas por abordaje endoscópico, 283 (61\%) correspondieron a cirugía endoscópica funcional y 156 (34\%) a dacriocistorrinostomía endoscópica.

De los procedimientos de faringe y laringe, la primera de estas áreas resultó ser la más prevalente, alcanzando 3.562 intervenciones, de las cuales $3.490(98 \%)$ correspondieron a cirugía adenoamigdaliana. Por su parte, de las 274 inter- venciones laríngeas, 177 (65\%) correspondieron a microcirugía laríngea.

En relación a la cirugía endoscópica de la vía aereodigestiva superior, se realizaron 370 procedimientos, entre los cuales 125 (34\%) correspondieron a esofagoscopias y $64(17 \%)$ a broncoscopías, siendo éstas mayoritariamente diagnóstico-terapéuticas ante la sospecha de cuerpo extraño. Por su parte, $96(26 \%)$ procedimientos correspondieron a panendoscopías, realizadas predominantemente en el contexto de diagnóstico y etapificación de patología oncológica de cabeza y cuello.

Respecto a la patología de cabeza y cuello, se realizaron 708 intervenciones, de las cuales 370 (52\%) correpondieron a traqueostomías, 43 (6\%) a parotidectomías y $34(5 \%)$ a tiroidectomias.

\section{DISCUSIÓN}

En nuestro centro se desarrolla una amplia y variada gama de procedimientos quirúrgicos

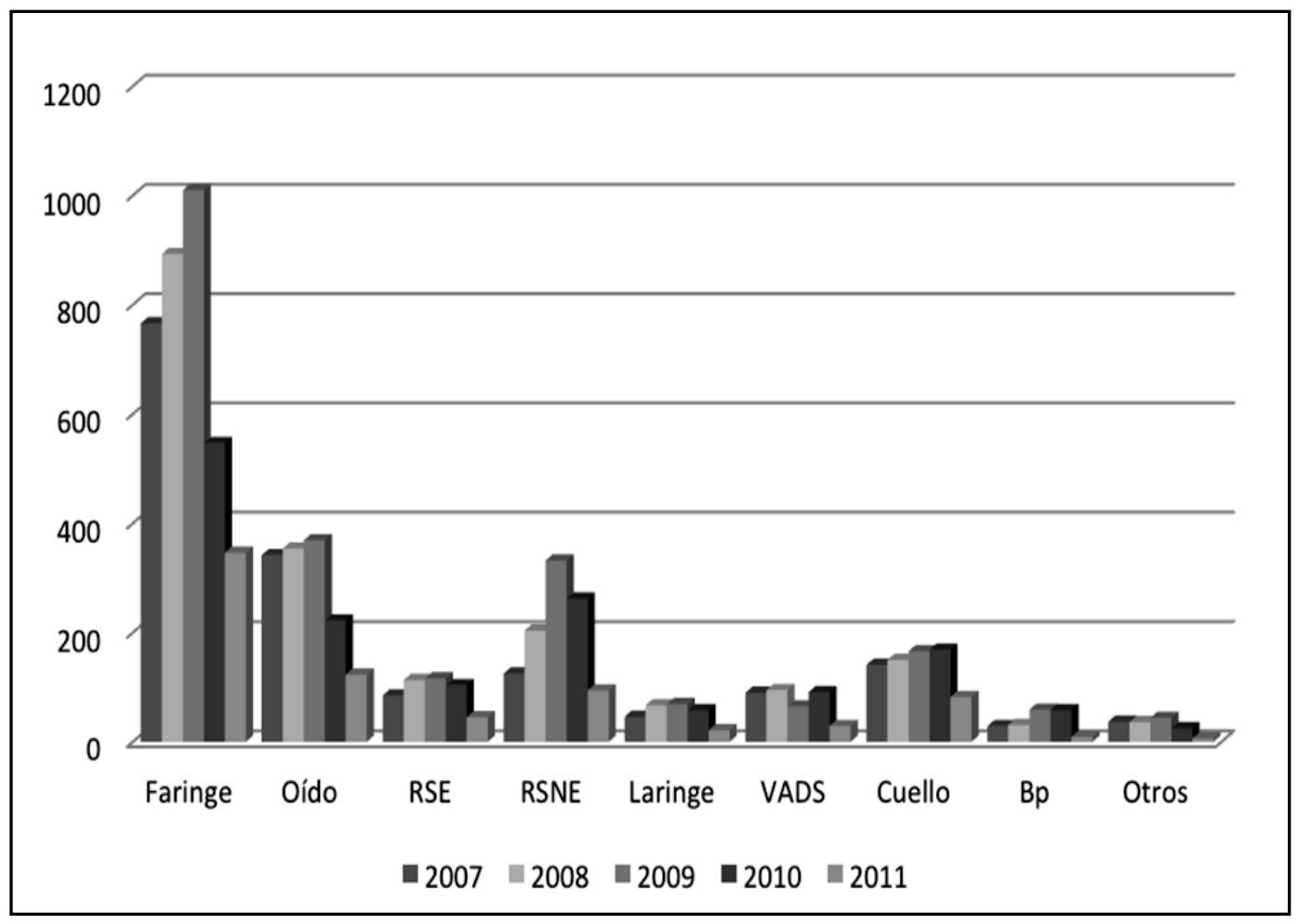

Figura 3. Distribución por área quirúrgica: Variación anual. 
otorrinolaringológicos, siendo la cirugía adenoamigdaliana la más prevalente durante el periodo estudiado. La práctica de cirugía endoscópica rinosinusal es frecuente en nuestro centro y de mantenerse la proyección actual, su importancia relativa será aún mayor en un futuro próximo.

Tanto el rendimiento quirúrgico como el índice de ocupación de camas del Servicio de Otorrinolaringología, está por sobre la media de nuestro centro hospitalario, lo que se debe en parte a la práctica de métodos mínimamente invasivos y el creciente desarrollo de la cirugía endoscópica, permitiendo la factibilidad de realizar cirugía mayor ambulatoria 0 en su defecto, hospitalización abreviada $^{3,4}$.

En los últimos años, se aprecia una tendencia a la disminución en la frecuencia de la cirugía otológica y al aumento de la cirugía rinosinusal, lo cual encuentra correlato en publicaciones nacionales previas 5 .

El incremento en la cirugía de cabeza y cuello se entiende al considerar en forma conjunta el tanto el envejecimiento de la población que condicionará sin duda un incremento en la patología oncológica, así como la reciente incorporación oficial de este departamento a nuestro servicio. Así pues, es esperable que en revisiones futuras, la cirugía de cabeza y cuello adquiera una mayor relevancia porcentual entre las demás áreas quirúrgicas desarrolladas por nuestro servicio ${ }^{7}$.

Por su parte, el descenso observado durante 2010 en la cirugía adenoamigdaliana se explica por la pérdida en camas quirúrgico-pediátricas sufrida por nuestro hospital como resultado del terremoto del 27 de febrero de 2010. Del mismo modo, la caída en el rendimiento quirúrgico observado en febrero de 2010 se explica por la pérdida de uno de los pabellones otorrinolaringológicos como consecuencia del mismo suceso. La recuperación del rendimiento quirúrgico en noviembre de 2010 se corresponde con la instauración de un programa de compra de servicios que ha permitido a nuestro servicio desarrollar cirugía mayor ambulatoria en centros privados.

El desarrollo de microcirugía laríngea se ha visto potenciado en los últimos años gracias a la adquisición por parte de nuestro servicio de un quipo de láser $\mathrm{CO2}$, el cual no sólo ha contribuido al desarrollo de nuevas técnicas y con esto a un mejor periodo posoperatorio y una mejor calidad de vida de nuestros pacientes, sino también a una formación más completa por parte de nuestros residentes.

Creemos que la cirugía de base de cráneo, así como la cirugía de cabeza y cuello, la primera de ellas no incluida y la segunda escasamente representada en la presente revisión, constituyen áreas de un potencial significativo en el futuro desarrollo de nuestra especialidad y esperamos a futuro presentar la exitosa experiencia que ha implicado su incorporación al quehacer cotidiano de nuestro servicio.

Finalmente, estamos conscientes de que si bien se han alcanzado logros sustantivos en la implementación de nuevas técnicas quirúrgicas por parte de nuestro servicio, tanto el perfeccionamiento de éstas, como la puesta en marcha de nuevas áreas aún en etapa de planificación, constituyen tareas continuas y aun con amplias expectativas de desarrollo.

Creemos también que la comunicación de los distintos logros de cada uno de los servicios de otorrinolaringología a lo largo del país, contribuye a incentivar la proyección de nuestra especialidad, tanto más en los profesionales nóveles, al tiempo que nos permite aprender de los aciertos y tropiezos de nuestros pares ${ }^{8}$. Estamos convencidos de que los diversos objetivos que cada uno de nuestros centros pueda plantearse, son factibles de alcanzar en la medida que exista la convicción y la adecuada optimización y gestión de los, muchas veces, escasos recursos con que podamos contar.

\section{BIBLIOGRAFÍA}

1. Ferrer F. Aspectos de la historia de la otorrinolaringología valenciana (1875-1950), Valencia, Instituto Médico Valenciano, 2002; 73-85.

2. PeñA A. Una breve historia de la invención del espejillo laríngeo y del espejo frontal. Rev Otorrinolaringol Cir Cabeza Cuello 2008; 68 (1).

3. Pérez G, Sandó B, Peña E, Gironés C. Major ambulatory surgery in otorhinolaryngology: a six-year experience, Hospital Clinicoquirúrgico «Comandante Manuel Fajardo», La Habana. Rev Cubana Cir 2008; 47 (3). 
4. Maluick A. Experiences of day care surgery in otolaryngology. Indian Journal of Otolaryngology and Head and Neck Surgery 2006; 58 (1).

5. Niklitschet E, Segovia T. Prevalencia de otitis media crónica en población escolar, sector occidente de Santiago. Cir Cabeza Cuello 1999; 59: 65-71.

6. Ulloa P, Urra A, Valenzuela A, Benavente J. Análisis de atención intrahospitalaria del Servicio de Otorrinolaringología, Hospital Guillermo Grant Benavemte de Concepción. Rev Otorriniolaringol Cir Cabeza Cuello 1999; 59: 93-100.

7. Ortega P, Urra A, Compan A. Experiencia clínica en tiroidectomía total. Rev Otorrinolaringol Cir Cabeza Cuello 2011; 71: 53-6.

8. Celedón C. Sentido del perfeccionamiento de la ORL. Rev Otorrinolaringol Cir Cab-Cue 2002; 62: 3-5. 